\title{
Nivel de satisfacción del servicio de agua potable en la ciudad de J uliaca, el caso de la urbanización J orge Chávez
}

\section{Level of satisfaction with the drinking water service in the city of J uliaca, the case of the J orge Chávez urbanization}

Recibido el 08/09/20 | Aceptado el 30/09/20

DOI: https://doi.org/10.47190/nric.v3i1.131

\author{
Juan Manuel Tito Humpiri \\ jtito@unaj.edu.pe - Universidad Nacional de J uliaca, J uliaca \\ Ricardo Quispe Quispe \\ ricardoquispe1994@gmail.com - Universidad Nacional de J uliaca, J uliaca \\ Abraham Melitón Contreras Vargas \\ econtreras@unaj.edu.pe - Universidad Nacional de Juliaca, Juliaca \\ Marcelino Reyes Casani Cruz \\ m.casani@unaj.edu.pe - Universidad Nacional de Juliaca, J uliaca \\ Edwin Huayhua Huamaní \\ ehuayhua@unaj.edu.pe -Universidad Nacional de Juliaca, Juliaca \\ Joly Zegarra Cáceres \\ jzegarracac@unsa.edu.pe - Universidad Nacional de J uliaca, Juliaca
}

\begin{abstract}
Resumen
El presente artículo científico tiene como objetivo; identificar el nivel de satisfacción del servicio de agua potable en la Urbanización de Jorge Chávez de la Ciudad de Juliaca, dicha investigación se realizó en el año 2019. El servicio en esta urbanización es proveído por la Entidad Prestadora de Servicios de Saneamiento SEDA Juliaca S.A. La satisfacción de los usuarios depende de algunos criterios como: la presión, calidad de atención y de servicio que ésta brinde. El método que se ha empleado es el descriptivo simple, se aplicó la encuesta como técnica y el cuestionario como instrumento, el mismo que está validada por la Superintendencia Nacional de Servicios de Saneamiento SUNASS, instrumento aplicado, en diferentes años, a las entidades prestadoras de servicios de saneamiento que están a su cargo, publicado posteriormente como Informe anual Benchmarking regulatorio; el tamaño de muestra fue de 215 usuarios, para medir la satisfacción de los usuarios cada pregunta fue medida con la escala de frecuencia de Likert. La hipótesis planteada afirma que es probable que el nivel de satisfacción en el servicio de agua potable en la urbanización Jorge Chávez de la ciudad de Juliaca sea bajo. En cuanto a los resultados; se ha demostrado que el nivel de satisfacción de los usuarios respecto a este servicio es ínfimo, esto debido a las pocas horas de cobertura, la presión es mínima y la atención no es oportuna al usuario. Se concluye con la ratificación de la hipótesis planteada además de demostrar que la cobertura del servicio de agua potable es de pocas horas lo que conlleva a la desconfianza. Los usuarios manifiestan que sus reclamos nunca fueron atendidos lo que ha generado desconfianza hacia la entidad prestadora de servicios de saneamiento en la ciudad de Juliaca. Finalmente, se ha determinado que el crecimiento poblacional hace que cada vez más usuarios queden insatisfechos con el servicio de agua potable. Las Empresas Prestadoras de Servicios rinden cuenta de su gestión a los organismos públicos, para ello utilizan indicadores de gestión exigidos desde diferentes instancias de gobierno, pero carecen de instrumentos para estimar la satisfacción de la población, que como se demuestra en el marco teórico, son necesarios para garantizar la sostenibilidad y gobernabilidad de las empresas.
\end{abstract}

Palabras claves: Servicio Público, Agua Potable, Satisfacción, Cobertura, Presión, Usuario.

Como citar: Tito-Humpiri, J.M., Quispe-Quispe, R., Contreras-Vargas, H.M., Casani-Cruz, M.R., Huayhua-Huamaní, E. \& Zegarra-Cáceres, J. (2020). Nivel de satisfacción del servicio de agua potable en la ciudad de Juliaca, el caso de la urbanización Jorge Chávez. ÑWWPRISUN - Revista de Investigación Científica, 3(1), 97-102. 


\section{Abstract}

This scientific article aims to; Identify the level of satisfaction with the drinking water service in the Jorge Chávez Urbanization in the City of Juliaca, this investigation was carried out in 2019. The service in this urbanization is provided by the Entidad Prestadora de Servicios de Saneamiento SEDA Juliaca S.A. User satisfaction depends on some criteria such as: the pressure, quality of care and service that it provides. The method that has been used is the simple descriptive one, the survey was applied as a technique and the questionnaire as an instrument, the same one that is validated by the National Superintendency of Sanitation Services - SUNASS, an instrument applied, in different years, to service providers of sanitation services that are in charge, subsequently published as Annual Regulatory Benchmarking Report; the sample size was 215 users, to measure user satisfaction, each question was measured with the Likert frequency scale. The hypothesis stated states that it is probable that the level of satisfaction in the drinking water service in the Jorge Chávez urbanization in the city of Juliaca is low. As for the results; It has been shown that the level of user satisfaction with this service is negligible, this due to the few hours of coverage, the pressure is minimal and the attention is not timely to the user. It concludes with the ratification of the hypothesis proposed, in addition to demonstrating that the coverage of the drinking water service is of a few hours, which leads to mistrust. Users state that their claims were never addressed, which has generated mistrust towards the en tity that provides sanitation services in the city of Juliaca. Finally, it has been determined that the population growth causes more and more users to be dissatisfied with the drinking water service. The Service Providers are accountable for their management to public bodies, for this they use management indicators required by different levels of government, but they lack instruments to estimate the satisfaction of the population, which as demonstrated in the theoretical framework, are necessary to guarantee the sustainability and governance of companies.

Keywords: Public Service, Drinking Water, Satisfaction, Coverage, Pressure, User.

\section{Introducción}

Durante las últimas tres décadas, el servicio de agua potable y saneamiento de América Latina ha experimentado sucesivas reformas con el objetivo de mejorar su desempeño. Sin embargo, la mayor parte de los países se encuentran lejos de alcanzar la universalidad de los servicios y restan esfuerzos para proveer niveles de calidad apropiados, en paralelo, subsisten problemas para el financiamiento de la operación e inversiones de los servicios.

En el informe final de evaluación 2013-2015 de la entidad prestadora de servicios de saneamiento de Juliaca al Organismo Técnico de la Administración de los Servicios de Saneamiento (OTASS), en la sección de acceso a los servicios literal indica que la cobertura de agua potable en el 2015 fue de $83.26 \%$, cifra superior a la obtenida en el año anterior (82.49\%). Esta cifra se ubica por debajo del promedio de las EPS municipales grandes $(90.82 \%)$ y del promedio del total de las EPS municipales (88. 81\%). asimismo, indica que la cobertura en horas, por día, ha disminuido de 8 horas en 2014 a 6.4 horas en el año 2015.

La cifra analizada se refleja en el descontento de los usuarios de agua potable en la urbanización Jorge Chávez y es el principal motivo por la cual se optó por desarrollar la investigación, asimismo, en dicha urbanización existe una atención inoportuna a los usuarios y por ende hay una insatisfacción de los usuarios. Por otro lado, se ha determinado que existe carencia de cobertura del servicio de agua potable, siendo efectivo el servicio solo 4 a 5 horas al día, y es motivo para dar a conocer la importancia de la calidad de servicio a la entidad prestadora y como esto afecta en la satisfacción de las necesidades principales de los usuarios del lugar, así puedan tener el conocimiento de la importancia de la calidad de servicios y buena atención al usuario. En virtud a la investigación se respondió la siguiente pregunta: ¿Cuál es el nivel de satisfacción del servicio de agua potable en la urbanización Jorge Chávez de la ciudad de Juliaca - 2019?, en tanto se demostró que el nivel de satisfacción de los usuarios es negativo, por tal motivo se elaborará propuestas de mejora a la entidad prestadora de servicios según el grado de satisfacción determinado.

Para Corrales (2003) en los últimos quince años se ha promovido la implantación de un nuevo arreglo institucional para la prestación de los servicios públicos por redes, orientado a introducir la dinámica del mercado como elemento ordenador del sector. Redefiniendo, la transformación ha marcado la agenda sectorial en casi todos los países de América Latina y el Caribe y a incluido un amplio proceso de cambio en el modelo de prestación del agua potable y saneamiento.

El servicio de Agua Potable tiene dos funciones: en primer lugar, abastecer el agua en condiciones sanitarias adecuadas al uso y consumo humano; el alejamiento y disposición de las aguas servidas y desechos a lugares convenientes para que se les pueda tratar y en segundo lugar asegurar el rehúso o vertido de los cauces naturales sin que esto ocasione el deterioro ecológico; preservando los recursos naturales. (INAP, 2009).

A través del libro; La calidad del agua potable en el Perú, la Superintendencia Nacional de Servicios de Saneamiento (Sunass), como entidad reguladora, supervisora y fiscalizadora del servicio que prestan las empresas de agua potable y alcantarillado sanitario o empresas prestadoras de servicios de saneamiento o empresas prestadoras (EPS), hace un llamado sobre la necesidad de desarrollar una gestión eficiente en el marco normativo del sector saneamiento. Así, la gestión de la calidad del agua potable se convierte en una de las expresiones más claras de la gestión empresarial de las EPS que administran este recurso, de su integración y compromiso con el entorno, así como de su visión de desarrollo organizacional.

De acuerdo a las estadísticas del INEI (2007) el 31.2 $\%$ de los habitantes del distrito de Juliaca tiene entre 15 y 29 años de edad. Asimismo, la tasa de 
crecimiento está proyectada en $2.68 \%$ al año, superior al $2.52 \mathrm{n} \%$ de la región de Puno y muy por encima del $1.6 \%$ del promedio del país; lo anterior podría representar una ventaja por el rápido crecimiento en términos de mano de obra y emprendimiento. Sin embargo, también significa dificultades para cubrir las necesidades de vivienda y saneamiento básico si se considera que solamente el $42.81 \%$ de la población cuenta con servicios de agua potable.

En el Perú, la realidad sobre la insatisfacción del usuario respecto a la calidad del servicio brindado por las EPS, representa un problema constante dentro de nuestra sociedad, la cual exige el cumplimiento de un derecho que le es consustancial a su condición de ser humano, tal como lo es el derecho a la prestación efectiva del servicio de agua potable, como expresión directa de un deber del Estado de procurar mejores condiciones de vida a sus ciudadanos.

\section{Agua potable y su importancia}

El agua de consumo inocua (agua potable), no ocasiona ningún riesgo significativo para la salud cuando se consume durante toda una vida, teniendo en cuenta las diferentes vulnerabilidades que pueden presentar las personas en las distintas etapas de su vida. Las personas que presentan mayor riesgo de contraer enfermedades transmitidas por el agua son los lactantes y los niños de corta edad, las personas debilitadas o que viven en condiciones antihigiénicas y los ancianos. El agua potable es adecuada para todos los usos domésticos habituales, incluida la higiene personal. No obstante, puede necesitarse agua de mayor calidad para algunos fines especiales, como la diálisis renal y la limpieza de lentes de contacto, y para determinados usos farmacéuticos y de producción de alimentos. (OMS, 2006)

El agua potable es reconocida por los clientes como el servicio público más importante, tiene un alto valor para la vida, la higiene, la salud y proporciona comodidad en el hogar.

El agua potable es esencial para la vida del ser humano. Es el líquido más importante de la naturaleza sin el cual no podríamos vivir. El agua potable nos ayuda a estar sanos, a hacer la digestión, mantiene la musculatura en buen estado, actúa refrigerando o calentando el cuerpo y ayuda a transportar el oxígeno entre las células de nuestro cuerpo. El planeta tierra tiene un promedio de $70 \%$ de agua, pero casi en su totalidad es agua salada. La misma no es buena para el consumo del hombre o los animales, así como para la agricultura o las industrias. El agua apta para el consumo es el agua dulce, pero es bastante escasa. Sólo el $3 \%$ del agua de la tierra es potable, y la mayoría aparece en forma de hielo en los polos terrestres. Por eso es muy importante conservar limpia el agua potable y detener la contaminación. Se hace urgente el cuidado del agua potable con el fin de evitar enfermedades que alcancen al ser humano, a los animales o a la agricultura en general.

Agüero y Barrios (2008) definen como agua potable aquella que cumple con los requerimientos de las normas y reglamentos nacionales sobre calidad del agua para consumo humano y que básicamente atiende a los siguientes requisitos: libre de microorganismos que causan enfermedades; libre de compuestos nocivos a la salud; aceptable para consumo, con bajo contenido de color, gusto y olor aceptables; y sin compuestos que causen corrosión o incrustaciones en las instalaciones sanitarias.

Según Jouravlev en los últimos 30 años el sector de agua potable y saneamiento en América Latina ha experimentado reformas sucesivas y de diferente naturaleza. La mayoría de los procesos de transformación han presentado características comunes tales como la descentralización, en muchos casos a nivel más bajo posible, o sea, municipal; la separación institucional entre las funciones de formulación de políticas sectoriales de regulación económica y de operación de los sistemas, incluida la creación de entes especializados de regulación y control de la prestación; y la participación privada, y más recientemente, en varios casos, salida de operadores privados internacionales y la reversión de la prestación pública. Para convertir el agua natural en otra apta para el consumo humano se requieren procesos, tecnología, recursos humanos y económicos para garantizar la sostenibilidad de los procesos de producción y distribución de agua potable, recolección, tratamiento de aguas residuales y disposición final de excretas, todas las actividades empresariales son reguladas, supervisadas y fiscalizadas por la Superintendencia Nacional de Servicios y Saneamiento - SUNASS, que ha desarrollado instrumentos de participación y transparencia con énfasis en el uso de los fondos públicos, y que aún no incluyen indicadores de satisfacción de la población en relación a la calidad de los servicios de agua potable saneamiento. A pesar de que el sector de agua potable y saneamiento ha sido objeto de profundas reformas, la región en su conjunto presenta aún importantes déficits. La mayor parte de los países se encuentran lejos de alcanzar la universalidad de los servicios de agua potable y saneamiento, a lo que se suma un déficit aún más alto de la materia de tratamiento de aguas servidas. En algunos casos también restan esfuerzo para proveer agua potable y niveles de calidad de prestación apropiados. Asimismo, subsisten problemas de financiamiento y no se han resuelto las carencias que afectan a la población de bajos recursos.

\section{Servicio}

Según Sangüesa (2006) un servicio es el resultado de llevar a cabo necesariamente al menos una actividad en la interfaz entre el proveedor y el cliente $y$ generalmente es intangible; es un conjunto de actividades que buscan satisfacer las necesidades de un cliente. Los servicios incluyen una diversidad de actividades que se pueden planificar desempeñadas por un gran número de personas (funcionarios, empleados, empresarios) que trabajan para el Estado (servicios públicos) o para empresas particulares (servicios privados); entre estos pueden señalarse los servicios de: electricidad, agua potable, limpieza, teléfono, telégrafo, correo, transporte, educación, internet, sanidad, asistencia social, etc. Se define un marco donde las actividades se desarrollarán con la idea de fijar una expectativa en el resultado de estas. Es el equivalente no material de un bien. Un servicio se diferencia de un bien en que el primero se consume y se desgasta de manera brutal puesto que la economía social nada tiene que ver con la política moderna; es muy importante señalar que la economía 
nacional no existe siempre en el momento en que es prestado.

\section{Satisfacción del usuario}

Calva (2009) afirma que "La satisfacción (resultados positivos) de una necesidad de información a través del comportamiento informativo, lleva a pensar en la evaluación de las fuentes y recursos utilizados por el sujeto para obtener dicha satisfacción".

Según, Álvarez (2012) el cliente percibe que el servicio supera lo esperado en cuanto a la dimensión; los clientes perciben que el servicio es peor al esperado en relación a las transacciones de venta, es decir el tiempo de espera para acudir a la cancelación en las cajas de salida.

Esto significa que las empresas privadas o públicas deben de tener una calidad de servicio hacia los usuarios, cuando un proveedor de servicios satisface las expectativas del cliente, tendremos la seguridad de que el usuario sienta comodidad y por parte del proveedor del servicio estará conforme económicamente, toda vez que no generará ningún problema.

\section{Niveles de satisfacción}

Para Thompson (2005) luego de realizada la compra o adquisición de un producto o servicio, los clientes experimentan uno de éstos tres niveles de satisfacción:

- Insatisfacción: Se produce cuando el desempeño percibido del producto no alcanza las expectativas del cliente.

- Satisfacción: Se produce cuando el desempeño percibido del producto coincide con las expectativas del cliente.

- Complacencia: Se produce cuando el desempeño percibido excede a las expectativas del cliente.

Dependiendo el nivel de satisfacción del cliente, se puede conocer el grado de lealtad hacia una marca o empresa, por ejemplo: Un cliente insatisfecho cambiará de marca o proveedor de forma inmediata (deslealtad condicionada por la misma empresa). Por su parte, el cliente satisfecho se mantendrá leal; pero, tan solo hasta que encuentre otro proveedor que tenga una oferta mejor (lealtad condicional). En cambio, el cliente complacido será leal a una marca o proveedor porque siente una afinidad emocional que supera ampliamente a una simple preferencia racional (lealtad incondicional). Por ese motivo, las empresas inteligentes buscan complacer a sus clientes mediante prometer solo lo que pueden entregar, y entregar después más de lo que prometieron.

\section{La importancia de la satisfacción al cliente}

Sin clientes, las empresas de servicio no tendrían una razón por la cual existir. Es necesario que toda empresa de servicio mida y defina, la satisfacción de servicio. Para la identificación de los problemas que se presentan en el servicio o para la evaluación del avance de la empresa, es necesario esperar a la queja de los clientes. (Grande, 1996).
Si bien, existen diversos beneficios que toda empresa u organización puede obtener al lograr la satisfacción de sus clientes, éstos pueden ser resumidos en tres grandes beneficios que brindan una idea clara acerca de la importancia de lograr la satisfacción del cliente. (Tabla 1).

Tabla 1

La importancia de lograr la satisfacción del cliente

\begin{tabular}{ll}
\hline \multicolumn{1}{c}{ Beneficio } & \multicolumn{1}{c}{ Acción del cliente } \\
\hline Primer Beneficio & $\begin{array}{l}\text { El cliente satisfecho, por lo general, vuelve } \\
\text { a comprar. Por tanto, la empresa obtiene } \\
\text { como beneficio su lealtad y por ende, la } \\
\text { posibilidad de venderle el mismo u otros } \\
\text { productos adicionales en el futuro. }\end{array}$ \\
$\begin{array}{l}\text { El cliente satisfecho comunica a otros sus } \\
\text { Begundo }\end{array}$ & $\begin{array}{l}\text { experiencias positivas con un producto o } \\
\text { servicio. Por tanto, la empresa obtiene } \\
\text { como beneficio una difusión gratuita que el } \\
\text { cliente satisfecho realiza a sus familiares, } \\
\text { amistades y conocidos. } \\
\text { El cliente satisfecho deja de lado a la } \\
\text { competencia. }\end{array}$ \\
\hline Nercer Beneficio &
\end{tabular}

En síntesis, toda empresa que logre la satisfacción del cliente obtendrá como beneficios:

La lealtad del cliente (que se traduce en futuras ventas); difusión gratuita (que se traduce en nuevos clientes) y una determinada participación en el mercado.

\section{Materiales y métodos}

La investigación tiene un enfoque cuantitativo, método descriptivo cuyo propósito es medir el nivel de satisfacción del servicio de agua potable en la urbanización Jorge Chávez de la ciudad de Juliaca en el año 2019, lo cual se ha realizado con la aplicación de una encuesta de satisfacción a través del instrumento de recolección de datos como es el cuestionario, el mismo que posee con 14 ítems de los cuales 7 están referidos al servicio de agua potable y los restantes a la atención al usuario que presta la entidad prestadora de servicios.

La población total con conexión de agua potable en la urbanización Jorge Chávez de la Ciudad de Juliaca es de 488 usuarios. De dicho número de elementos de la población, se determinó el tamaño de la muestra con la siguiente fórmula:

$$
\mathrm{n}=\frac{488(0.5)^{2}(1.96)^{2}}{(488-1)(0 \cdot 05)^{2}+(0.5)^{2}(1.96)^{2}}
$$

De acuerdo a la fórmula, el resultado es de la muestra es de 215 usuarios que viven en la urbanización Jorge Chávez y que cuentan con el servicio de agua potable. 


\section{Resultados y discusión}

En la tabla 2 se puede apreciar el nivel de satisfacción del servicio de agua potable

Tabla 2.

Satisfacción del servicio de agua potable

\begin{tabular}{clrr}
\hline Valoración & Respuesta & № & \multicolumn{1}{c}{$\%$} \\
\hline $\mathbf{1}$ & Deficiente & 28 & $13 \%$ \\
$\mathbf{2}$ & Mala & 113 & $53 \%$ \\
$\mathbf{3}$ & Regular & 62 & $29 \%$ \\
$\mathbf{4}$ & Buena & 11 & $5 \%$ \\
$\mathbf{5}$ & Muy Buena & 1 & $0 \%$ \\
\hline Total & & 215 & $100 \%$ \\
\hline
\end{tabular}

Los resultados sistematizados en la tabla demuestran que más del $50 \%$ consideran el servicio como deficiente o malo, así mismo el $29 \%$ de entrevistados considera que el servicio es regular y solo el $5 \%$ considera que el servicio es bueno.

Cumplido la sistematización de los resultados y que han sido demostrados anteriormente, es necesario discutir estos resultados con los antecedentes y marco teórico; a continuación, presentamos la discusión de resultados de la presente investigación.

Los resultados manifiestan que el nivel de satisfacción en general es malo o bajo 53\%, llegando a concordar con Ponce (2018) ya que sus resultados demuestran que el $82.8 \%$ está muy insatisfecho o insatisfecho con el agua potable y desagüe que reciben por parte de esta empresa, la diferencia porcentual entre la percepción $(44.08 \%)$ y expectativas $(85.08 \%)$ por el producto es de alrededor del $41 \%$, con lo cual se puede determinar que hay una satisfacción ínfima por parte de los usuarios en relación al producto brindado por la Empresa Prestadora de Servicios de Juliaca. Finalmente, los resultados concuerdan con Angulo \& Peralta (2016) quienes concluyen que los usuarios encuestados en su mayoría respondieron que a veces cumple sus necesidades debido a que no tienen el agua todo el día y llega poca agua a sus domicilios.

\section{Conclusiones}

El agua potable que reciben los pobladores de la urbanización Jorge Chávez del distrito de Juliaca posee olor, color o sabor anormal, con ello se demuestra que la entidad no estaría cumpliendo en proveer agua potable apto para el consumo humano tal como lo señala la Superintendencia Nacional de Servicios de Saneamiento.

El nivel de satisfacción es ínfimo con relación a servicio que presta la Empresa Prestadora de Servicio de Saneamiento SEDA Juliaca S. A., con ello se ha ratificado la hipótesis general planteado en la investigación. Asimismo, se ha probado que la cobertura del servicio de agua potable es de pocas horas en la urbanización por lo que el servicio de agua potable no es fiable ni oportuno para los usuarios.

Los reclamos realizados por los usuarios en la mayoría de los casos no tuvieron una respuesta inmediata, provocando que los usuarios tengan desconfianza hacia la entidad prestadora de servicios de saneamiento.

El crecimiento poblacional hace que cada vez más usuarios queden insatisfechos con el servicio de agua potable, para ello la entidad debe proponer políticas sectoriales para solucionar la insatisfacción de los usuarios. 


\section{Referencias bibliográficas}

Álvarez, C. G. (2012). Satisfacción de clientes y usuarios con el servicio ofrecido en redes de supermercados gubernamentales. Universidad Católica Andrés Bello. Venezuela.

Angulo, K., \& Peralta, V. (2016) Nivel de satisfacción de los usuarios del servicio de agua potable de la ciudad de Cajamarca, 2015 (Tesis de pregrado) Universidad Privada del Norte, Cajamarca.

Corrales, M. E. (2003) Gobernabilidad de los servicios de agua potable y saneamiento en América Latina. Brasil.

Calva J. J. (2009). satisfacción de usuarios: la investigación sobre necesidades de información. México: UNAM.

Gutiérrez, J. (2016) Calidad de los servicios de saneamiento básico y su relación con la satisfacción del usuario en el distrito de J uanjui - provincia de Mariscal Cáceres 2016 (Tesis de maestría) Universidad César Vallejo, TarapotoPerú.

Jouravlev, A. (2004), "Participación privada sustentable: perspectivas y experiencias", seminario internacional " $\mathrm{Rol}$ del regulador de agua potable y saneamiento en el siglo XXI: retos y oportunidades", Comisión Económica para América Latina y el Caribe (CEPAL), colección documentos de proyectos, LC/W.332, Santiago de Chile (disponible en internet: http://www.eclas.org).

Instituto Nacional de Administración Pública (2009) Guías Técnicas Municipales. Recuperado de http://www.inafed.gob.mx/work/models/inafed/ Resource/322/1/images/guias_inap.pdf

Instituto Nacional de Estadística e Informática, (2004). Perú: compendio Estadístico 2003. Lima: INEI.

Lampoglia, T., Agüero, R. \& Barrios, C. (2008). Orientaciones sobre agua y saneamiento para zonas rurales. Recuperado de: http://www.bvsde.paho.org/bvsacg/guialcalde/ 2sas/d21/019 SER OrientacionesA\&Szonasr urales/Orientaciones\%20sobre\%20A\&S\%20p ara\%20zonas\%20rurales.pdf

Organismo Mundial de la Salud (2006) Guías para la calidad del agua potable. Volumen 1. Recuperado de: https://www.who.int/water_sanitation_health/d wq/gdwq3_es_fulll_lowsres.pdf

Ortega, M. (2016), La percepción de los usuarios del servicio de agua potable en Xalapa (Proyecto de intervención). Universidad Veracruzana, México.

Ojeda, L. \& Quispe, V. (2017) Calidad de servicio asociado con la satisfacción del cliente en la empresa El Bazar de Charly's de la ciudad de Juliaca-Puno 2017 (Tesis de Pregrado). Universidad Peruana Unión, Lima.

Organismo Técnico de la administración de los servicios de saneamiento. (2016) Entidad prestadora de servicios de saneamiento de Juliaca, informe final de evaluación 2013-2015 (informes finales de evaluación) recuperado de http://www.otass.gob.pe/publicaciones/informe s/informe-de-evaluacion/informe-deevaluacion-de-eps-periodo-2013- 2015/informes-finales-de-evaluacion-3/2086eps-sedajuliaca-1/file.html

Pastor, O. (2014) Evaluación de la satisfacción de los servicios de agua y saneamiento urbano en el Perú: De la imposición de la oferta a escuchar a la demanda (tesis de maestría). Pontificia Universidad Católica del Perú, Lima.

Patrick, P. (2019). La calidad del servicio de agua potable de EMAPA SAN MARTín S.A. y su influencia en la satisfacción de los usuarios de sector parte alta del distrito de Morales, periodo enero a junio 2016 (Tesis de maestría). Universidad César Vallejo, Tarapoto-Perú.

Sanguesa, S. M. (2006). Teoría y Práctica de la Calidad. España: Paraninfo.

Thompson, I. (2005) "La Satisfacción del Cliente: Conozca cuáles son los beneficios de lograr la satisfacción del cliente, cómo definirla, qué elementos la componen y cuál es la fórmula para determinar el nivel de satisfacción del cliente. Bolivia.

Velasco, V. (2011). La Calidad del Servicio y la Satisfacción de los Clientes del Comisariato FECOS de la ciudad de Salcedo (tesis de pregrado). Universidad Técnica de Ambato, Ecuador.

Vidangos, W. (2017) Estrés laboral (Síndrome de Burnout) y efectos en la satisfacción en el trabajo en las empresas prestadoras de servicios de saneamiento básico de la región puno; 2016 (Tesis de maestría). Universidad Nacional del Altiplano, Puno-Perú.

Estudio Tarifario: Determinación de la fórmula tarifaria, estructura tarifaria y metas de gestión aplicable a la entidad prestadora saneamiento de Juliaca - EPS SEDAJULIACA S.A. Recuperado de https://sedajuliaca.com/wpcontent/uploads/2019/07/estudiotar.pdf

Memoria Anual 2018 de EPS SEDAJULIACA S.A. recuperado de https://sedajuliaca.com/wpcontent/uploads/2019/07/memoria2018.pdf

Código de buen gobierno corporativo de la Entidad Prestadora de Servicios de Saneamiento de Juliaca - EPS SEDAJULIACA S.A. que corresponde al año 2018. Recuperado de https://sedajuliaca.com/wpcontent/uploads/2019/07/cod_buen_gobierno 2018.pdf

PLAN ESTRATÉGICO SECTORIAL MULTIANUAL (PESE M) 2016 - 2021, Ministerio de Vivienda, Construcción y Saneamiento. Recuperado de https://sedajuliaca.com/wpcontent/uploads/2019/07/pesem.pdf

Superintendencia Nacional de Servicio de Saneamiento. Portal web, 2020. Recuperado de

https://www.sunass.gob.pe/websunass/index. php/sunass/quienes-somos 